\title{
Total Ankle Replacement Requiring Distal Tibiofibular Arthrodesis in a Dog
}

\author{
Neil J. Burton ${ }^{1}$ Maciej Krukowski ${ }^{1}$ \\ ${ }^{1}$ Wear Referrals Veterinary Hospital, Bradbury, Stockton-On-Tees, \\ United Kingdom \\ VCOT Open 2021;4:e12-e19.
}

\begin{abstract}
Address for correspondence Neil Burton, BVSc, MResCVR, DSAS (Orth), CertSAS, PGCert(HE), FHEA FRCVS, Wear Referrals Veterinary Hospital, Bradbury TS21 2ES, Stockton-on-Tees, United Kingdom (e-mail: neil.burton@wear-referrals.co.uk).
\end{abstract}

\begin{abstract}
Keywords

- tibiofibular arthrodesis

- canine ankle replacement
\end{abstract}

This case report describes distal tibiofibular arthrodesis as a technique for achieving increased confluent bone support for the placement of oversized arthroplasty components for talocrural arthroplasty in an 18-month-old Labrador Retriever with talocrural osteoarthritis secondary to talar osteochondrosis. Computed tomography assessment for suitability for BioMedtrix canine ankle replacement surgery revealed the tibia to be undersized relative to the tibial component. Distal tibiofibular arthrodesis was performed to increase lateral bone support to permit placement of an otherwise oversized prosthesis. Subjective assessment of outcome with owner Liverpool Osteoarthritis in Dogs questionnaire to 6 months postoperatively as well as radiological assessment to 4 months postoperatively documented significant improvement in lameness in the operated limb with no complications. Distal tibiofibular arthrodesis is a means by which to achieve increased bone support prior to BioMedtrix canine total ankle replacement surgery. The surgical technique described herein permitted placement of an oversized talocrural prosthesis in this patient with good clinical function. This technique may permit use of this arthroplasty system in otherwise undersized patients until such a time that smaller implants are available from the manufacturer.

\section{Introduction}

The ankle joint bears the highest stress of all the extremity joints in $\operatorname{man}^{1}$ and it is estimated that $1 \%$ of the global population is affected by osteoarthritis of this joint. ${ }^{2}$ Where medical management is ineffective and recalcitrant pain is present, total ankle arthroplasty has been developed in humans over the past 30 years in an attempt to maintain ankle range of motion and function which are factors mitigated by arthrodesis; the latter of which may result in inferior function. ${ }^{3}$ Long-term review of newer generation system outcomes has found good to excellent results in $82 \%$ patients with two-component designs. ${ }^{4}$ Newer three-component designs which incorporate a mobile bearing or 'meniscus' on meta-analysis show a 5 -year prosthesis survival of $90.6 \%^{5}$

received

October 3, 2020

accepted after revision

December 18, 2020
DOI https://doi.org/

10.1055/s-0041-1723796.

ISSN 2625-2325.
Osteoarthritis of the talocrural joint is similarly described in dogs and is commonly associated with osteochondrosis of the talus. ${ }^{6,7}$ Canine talocrural hemiarthroplasty using a custom manufactured implant has been described. ${ }^{8}$ The first talocrural total arthroplasty; the BioMedtrix Canine Total Ankle Replacement (BCTAR, BioMedtrix, Whippany, New Jersey, USA) (-Fig. 1) was first implanted clinically in $2015^{9}$ and is a cementless semi-constrained cartridge developed utilizing the same drill-and-mill technology for bone preparation as the BioMedtrix TATE elbow. ${ }^{10}$ Design modifications to both the implant-bone interface fixation and surgical technique have evolved from those of the TATE elbow, including the use of hollow fixation posts that expand when bolts are placed to secure the implant and a starter and finisher milling arm to make final milling of the bone more

\section{(c) 2021. The Author(s).}

This is an open access article published by Thieme under the terms of the Creative Commons Attribution License, permitting unrestricted use, distribution, and reproduction so long as the original work is properly cited. (https://creativecommons.org/licenses/by/4.0/) Georg Thieme Verlag KG, Rüdigerstraße 14, 70469 Stuttgart, Germany 


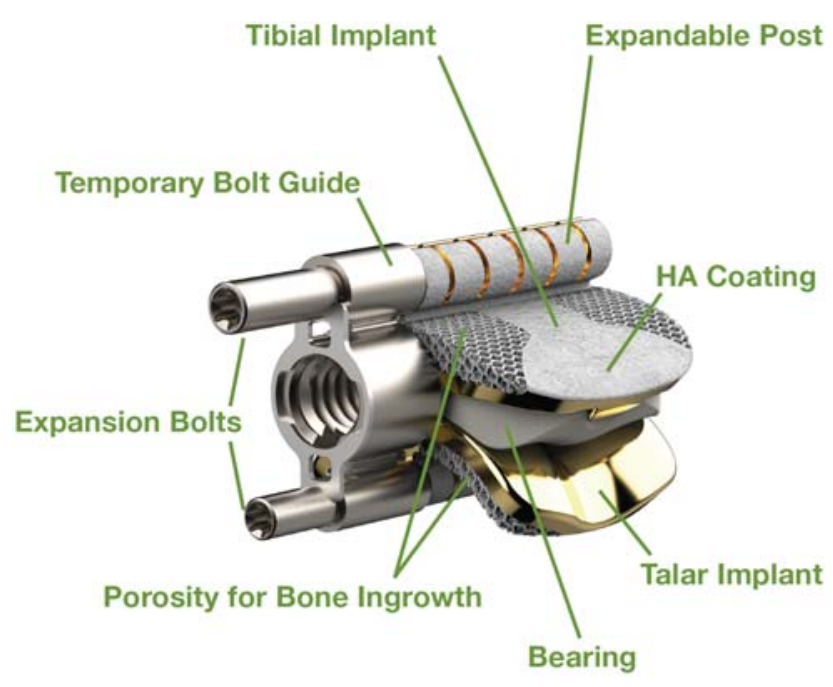

Fig. 1 Design features of the BioMedtrix Canine Total Ankle Replacement. The assembled cartridge consists of tibial and talar components with hydroxyapatite coated porous titanium ingrowth surfaces, titanium nitride articular surfaces (talar) and ultrahigh molecular weight polyethylene with vitamin E (tibial). Titanium expansion bolts are advanced into expandable posts to secure the implant. The temporary bolt guild is used to attach the prosthesis to an inserter. Image courtesy: BioMedtrix.

accurate. A phase II clinical trial is currently underway of the BCTAR. A published clinical series assessing outcome of this arthroplasty system is lacking, although abstract presentation of pilot clinical cases has been encouraging. ${ }^{9}$ Currently, only one size of this arthroplasty system, is available for use in dogs.

This case report describes distal tibiofibular arthrodesis and BCTAR surgery. Arthrodesis was performed to provide adjunctive bone support to a tibial component that was otherwise oversized for the patient to be able to proceed with BCTAR.

\section{Case Report}

An 18-month-old female neutered $33 \mathrm{~kg}$ Labrador Retriever presented with a 5-month history of progressive left pelvic limb lameness. Clinical examination by its veterinarian had revealed left hock soft tissue swelling, pain on palpation and joint effusion. Radiographs had been performed that had revealed talocrural osteoarthritis, pronounced medial soft tissue thickening, effusion and widening of the medial aspect of the talocrural joint suggestive of osteochondrosis of the medial ridge of the talus (-Fig. 2A). Arthrocentesis of the joint had been performed that had revealed moderate macrophagic and mononuclear inflammation (nucleated cell count of 2140 cells $/ \mu \mathrm{L}$, protein of $54 \mathrm{~g} / \mathrm{L}$ ) consistent with chronic osteoarthritis. The dog had been prescribed meloxicam (Metacam; Boehringer Ingelheim, United Kingdom) $0.1 \mathrm{mg} / \mathrm{kg}$ every 24 hours for 3 months but there had been minimal improvement in lameness on this medication.

Physical examination revealed $6 / 10,{ }^{11}$ left pelvic limb lameness with moderate pain on full flexion of the left hock joint, effusion and periarticular soft tissue thickening.

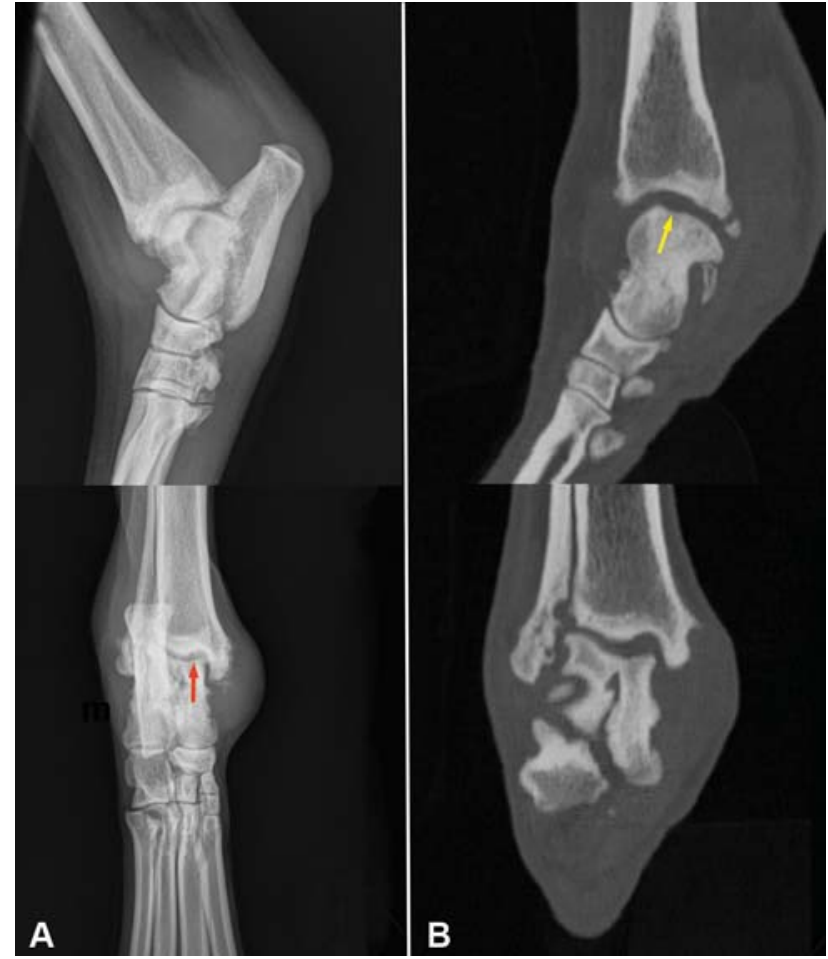

Fig. 2 (A) Radiological changes in the left hock joint-there is soft tissue thickening of the talocrural joint, especially medially and osteophytosis. Subjectively, the medial portion of the tibiotalar joint is widened (red arrow). (B) Computed tomographic changes in the left hock joint-sclerosis of the distal tibia and talus is present as well as osteophytosis, medial tibiotalar joint space widening and a defect in the medial talar ridge on the sagittal view (yellow arrow).

Range of motion of the joint, as assess with goniometry, ${ }^{12}$ was 70 degrees flexion and 165 degrees extension. The remainder of the clinical examination was unremarkable.

Computed tomography (CT) of the left hock ( - Fig. 2B) was performed with sedation; medetomidine (Sedator, Dechra Veterinary Products, United Kingdom) $10 \mu \mathrm{g} / \mathrm{kg}$, butorphanol (Torbugesic, Zoetis, United Kingdom) $0.2 \mathrm{mg} / \mathrm{kg}$. This revealed osteoarthritis of the talocrural joint with an osteochondral defect of the medial trochlear ridge consistent with osteochondrosis; fragmentation of talar ridge bone was not identified.

Clinical findings were reviewed with the owner of the dog. Continued conservative management, pantarsal arthrodesis and BCTAR were discussed as management options for this case with the current evidence base and fair disclosure for each therapeutic option being presented to the owner. The client was keen to pursue BCTAR if possible, in an effort to both preserve joint motion and improve patient lameness.

Review of the CT with reference to suitability of the case for BCTAR was performed. The dimensions of the tibial and talar components were assessed in relation to the tibial and talar bone ( - Fig. 3 ). This revealed the tibial component to be wider that the width of the distal tibia in the dorsal plane when accommodating a medial malleolar osteotomy to gain access to the joint such that placement of the prosthesis would have to encroach in the distal tibio-fibular osseous gap and into $\sim 50 \%$ of the fibular width. Concerns regarding 

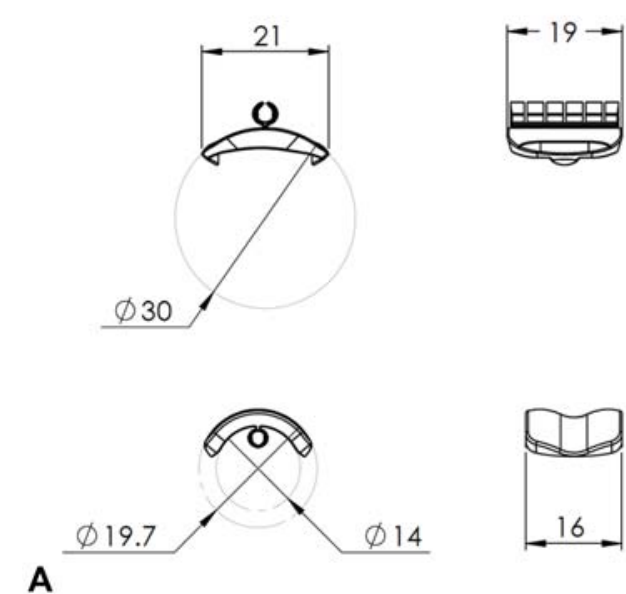

Fig. 3 Implant templating. (A) Mediolateral and craniocaudal dimensions of the tibial and talar components, measurements in millimetres. (B) dorsal slice computed tomography (CT) showing the planned medial malleolar osteotomy (red), tibial (yellow) and talar (blue) components. The tibial component is oversized relative to the tibia such that it crosses into the tibiofibular syndesmosis and $50 \%$ of the width of the fibula. (C) Sagittal slice CT showing tibial (yellow) and talar (blue) components. Image courtesy (A): BioMedtrix.

placement of the implant in this way related to a portion of the lateral aspect of the tibial component not being supported by bone, being supported by both the tibia and fibular risking micromotion along the tibial component-bone interface and as such aseptic loosening, as well as the risk of distal fibular fracture and associated lateral collateral ligament insufficiency. As such, performing distal tibiofibular arthrodesis prior to arthroplasty was proposed in an effort to improve confluent trabecular bone stock for support of the tibial component.

\section{Distal Tibiofibular Arthrodesis}

The patient returned for arthrodesis surgery 2 weeks later. Surgery was performed with general anaesthesia (medetomidine $5 \mu \mathrm{g} / \mathrm{kg}$ intravenous [IV], methadone $0.2 \mathrm{mg} / \mathrm{kg}$ IV, propofol -Propofol Plus, Zoetis, United Kingdom) and inhalational isoflurane (Isoflo, Zoetis, United Kingdom, in 100\% oxygen). Ultrasound-guided left sciatic nerve block $0.1 \mathrm{mg} / \mathrm{kg}$ levobupivicane (Chirocaine, Abbvie, UK), paracetamol $10 \mathrm{mg} / \mathrm{kg}$ IV (Perfalgan, Bristol-Myers Pharmaceuticals, United Kingdom), meloxicam $0.1 \mathrm{mg} / \mathrm{kg}$ IV (Metacam, Boehringer-Ingelheim, United Kingdom, cefuroxime, $20 \mathrm{mg} / \mathrm{kg}$ every 90 minutes) (Zinacef, Covis Pharmaceuticals, United States) were administered concurrently. The skin over the left ilial wing and left pelvic distal limb from stifle to digits was clipped and aseptically prepared and then sterilely draped for surgery.

An autogenous corticocancellous bone graft was harvested from the left ilial wing using an acetabular reamer (Universal Hip System, BioMedtrix, United States) via approach to the wing of the ilium. ${ }^{13}$ Thereafter, a lateral approach to the distal fibular/lateral malleolus was performed. ${ }^{14}$ The deep digital flexor tendon caudal to and tendon of the peroneus longus cranial to the lateral malleolus were mobilized and retracted caudally and cranially respectively. A sharp periosteal elevator and spinal burr (Hall Surgairtome Two, Veterinary Instrumentation, United Kingdom) were used to scarify the bone and remove cartilage from the distal tibiofibular syndesmosis extending from the tibiofibular joint capsule both caudally and cranially as well as proximally over $3 \mathrm{~cm}$ ( - Fig. 4A). The graft was then packed in to both caudal and cranial tibiofibular recesses, and the soft tissues and skin were closed with 3.0 Monocryl and 4.0 Ethilon (Ethicon, Johnson \& Johnson, Belgium) respectively. The patient was discharged on oral meloxicam and the recommendation for light lead exercise only for 6 weeks.

Six weeks postoperatively the patient was admitted for left BCTAR. The day prior to this surgery the patient was sedated as previously described and CT of the left distal tibia/hock performed which, when compared with preoperative $\mathrm{CT}$, revealed evidence of progression of arthrodesis at the site of grafting ( $\mathbf{- F i g . ~ 4 B , ~ C ) . ~}$

\section{BioMedtrix Canine Total Ankle Arthroplasty}

The patient was anaesthetized, prepared and draped for surgery of the left hock with the aforementioned protocol. The left hock was instrumented as per a BioMedtrix video that had been distributed to all surgeons undertaking their phase II clinical trial.

Briefly, (and as illustrated in -Fig. 5), the patient was positioned in left lateral recumbency with the left pes on a Mayo stand. An approach to the medial malleolus and talocrural joint was performed with medial malleolar osteotomy ${ }^{14}$ with a sagittal saw (Collibri II, DePuy Synthes, United Kingdom), with care taken to avoid damage to the medial collateral ligaments. Inspection of the talus revealed a medial talar ridge osteochondral defect; no loose flap of cartilage or bone was present. Following visualization of the medial aspect of the talus, a calliper was used to define the central axis of rotation (COR) of the bone by placing the tip of one end in the center of the talus and the other tip to trace the circumference of the medial trochlear ridge. A hole was then drilled through the COR of the talus using a $2 \mathrm{~mm}$ drill bit following which the COR post was placed through this hole (-Fig. 5A). The alignment plate was slid over the COR and the 


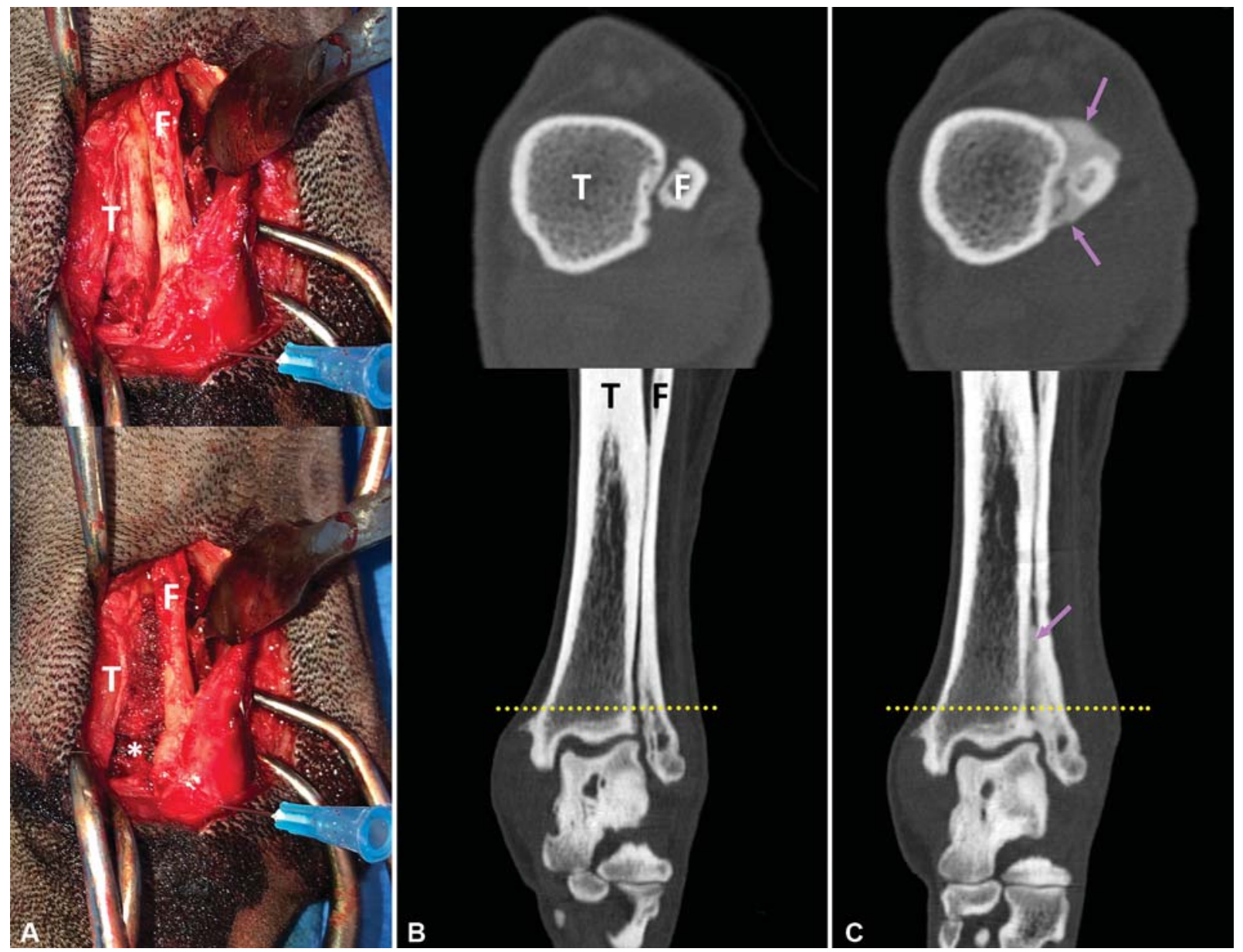

Fig. 4 (A) Intraoperative images showing the fibula (F) and tibia (T) prior to and following placement of the autogenous bone graft ${ }^{*}$ ). (B) Preoperative transverse and dorsal plane computed tomographic (CT) images ( $T=$ tibia, $F=$ fibula), the yellow dotted line denotes the level of the transverse CT slice. (C) Six weeks post arthrodesis transverse and dorsal place CT images, new bone is evident bridging the fibula to the tibia (purple arrows).

tibial extension of the alignment plate aligned with the anatomical axis of the tibia. The alignment plate was then attached to the tibia with two $2.4 \mathrm{~mm}$ Ellis pins placed through two drill cannulae, which were locked in the alignment plate with set screws (-Fig. 5B). The Ellis pins were then locked to the drill cannulae with set screws. The hock was then flexed such that a $2.4 \mathrm{~mm}$ Ellis pin placed through the calcaneal hole and associated drill cannula in the alignment plate bisected the calcaneus in the dorsoplantar plane, this pin being placed in the calcaneus. An identical pin was placed through the talar hole and associated drill cannula in the alignment plate into the base of the talus; all set screws were tightened. The $7 \mathrm{~mm}$ starter end mill was then attached to the magnetic milling arm, a drill stopper was used to define the length of milling arm available to mill, corresponding to the width of the ankle replacement trial implant. Gelpi and small Hohmann retractors were used to retract the soft tissues away from the site of milling. The joint was then milled using 2 to $4 \mathrm{~mm}$ progressive depth advancements through the bone with each sequential arc of the milling arm until final depth was reached ( - Fig. 5C). Lavage and suction were used to cool the bone and remove bone debris. The
$8 \mathrm{~mm}$ finisher end mill was then attached to the magnetic milling arm, the drill stopper used identically and then the joint milled again with identical milling technique. Following milling, careful inspection for any bone fragments in the soft tissues was performed and any removed. The trial implant was then attached to the inserter and placed to check an appropriate depth of mill was achieved, assessed by the trial being level relative to the medial aspect of the talus. The drill guide was then attached to the alignment plate and a 4 and $2.5 \mathrm{~mm}$ drill bit used to drill the tibial and talar component fixation holes respectively. The rib-breaker was attached to the inserter and advanced into the tibial and talar holes, cutting through the ribs of bone between the holes and the milled region (-Fig. 5D). The cartridge implant was then attached to the inserter (-Fig. 5E). The tibial and talar fixation posts were aligned with the tibial and talar fixation holes and then the cartridge pushed into the prepared joint space. The inserter was then unscrewed from the cartridge, the set plate removed, the post guide removed and then the tibial and talar posts screwed in, expanding the tibial and talar component posts and locking each component into the bone. The Ellis pins and alignment plate were removed 


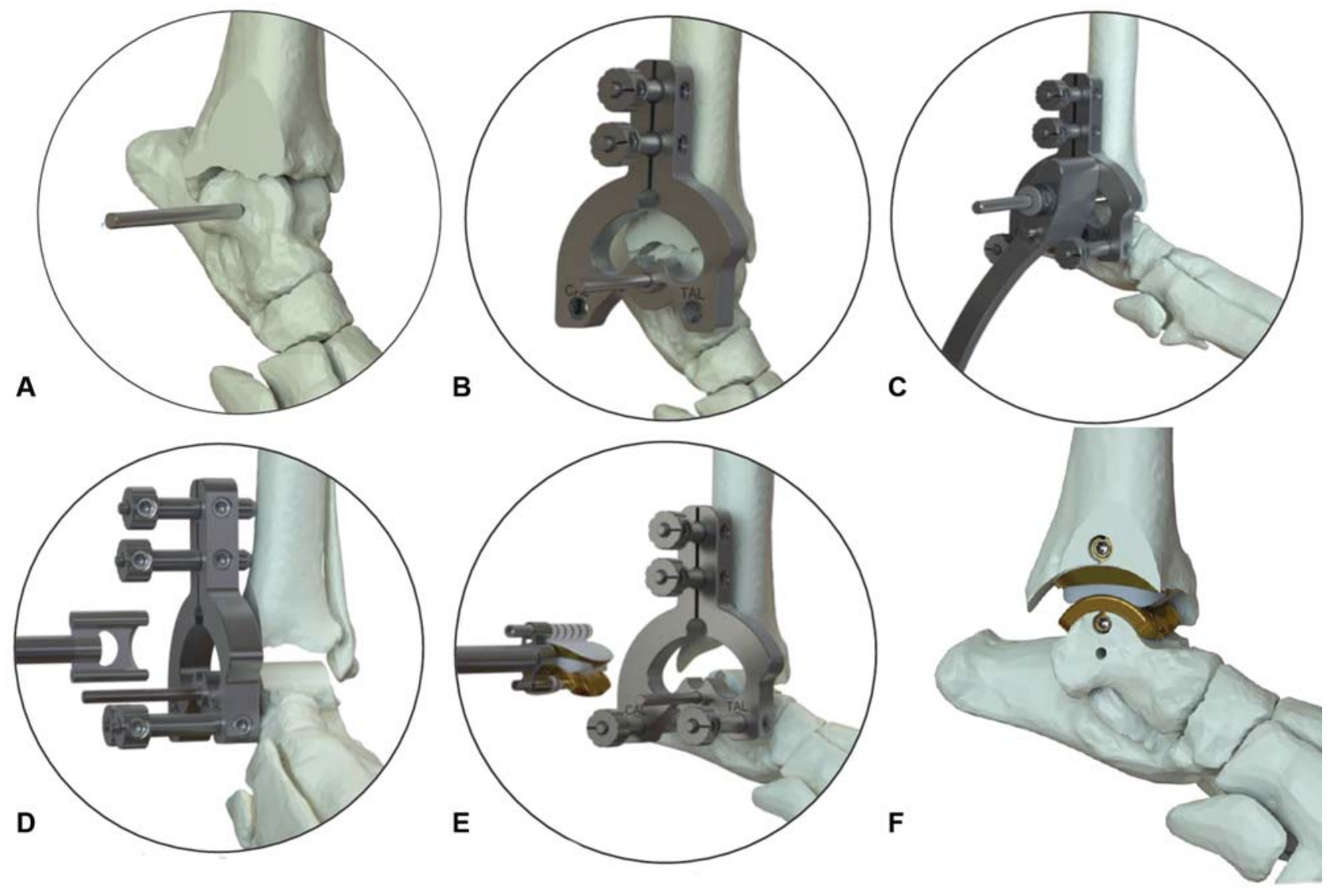

Fig. 5 Summary of the steps of bone preparation and implantation of the ankle prosthesis-see text for details. Image courtesy: BioMedtrix.

(-Fig. 5F). Range of motion was assessed, and any remaining osteophytes removed if they were impinging on the implant's range of motion (-Fig. 6). The medial malleolus was reduced back into position and held in compression against the tibia with two pairs of speed-lock bone holding forceps. A seven-hole $2.7 \mathrm{~mm}$ locking compression plate (De Puy Synthes, United Kingdom) was applied to the medial malleolus and distal tibia to secure the malleolus.

In addition to the standard implantation technique for the BCTAR, a skin incision was then made over the lateral malleolus and a nine-hole $2.0 \mathrm{~mm}$ locking compression plate applied to the lateral aspect of the fibula with the three proximal screws engaging the tibia concurrently. Medial and lateral subcutaneous soft tissues and skin were closed with 4-0 Monocryl and 4-0 Ethilon respectively. Postoperative radiographs were performed that showed appropriate implant placement and positioning (-Fig. 7).

The limb was placed in a light compression dressing for 48 hours. Postoperative analgesia (methadone $0.2 \mathrm{mg} / \mathrm{kg}$ IV every 4 hours for 24 hours, switching to buprenorphine, $0.02 \mathrm{mg} / \mathrm{kg}$ IV for 36 hours, paracetamol $10 \mathrm{mg} / \mathrm{kg}$ every 8 hours and meloxicam $0.1 \mathrm{mg} / \mathrm{kg}$ every 24 hours) was provided. A 7-day course of oral paracetamol $(10 \mathrm{mg} / \mathrm{kg}$ every 8 hours) and cephalexin (20mg/kg every 12 hours) and a 21 day course of meloxicam $(0.1 \mathrm{mg} / \mathrm{kg}$ every 24 hours $)$ were prescribed.

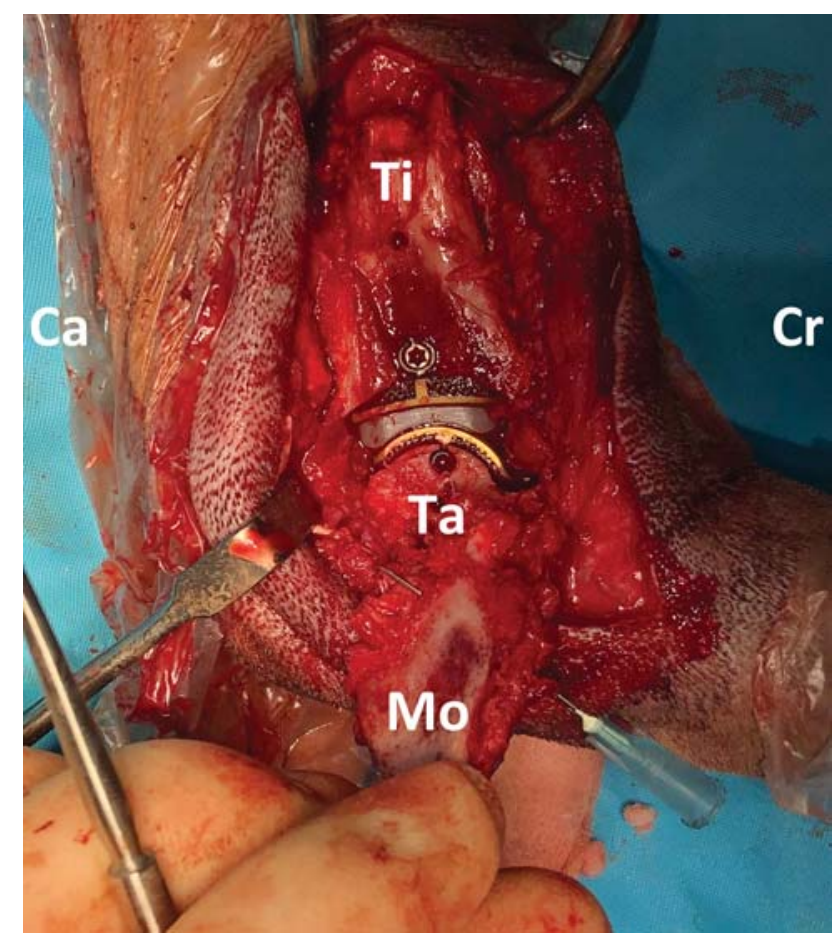

Fig. 6 Intraoperative view following placement of the prosthesis, advancement of the tibial and talar component expansion bolts and removal of the set plate. Ca, caudal; $\mathrm{Cr}$, cranial; Mo, medial malleolar osteotomy; Ti, tibia, Ta, talus. 


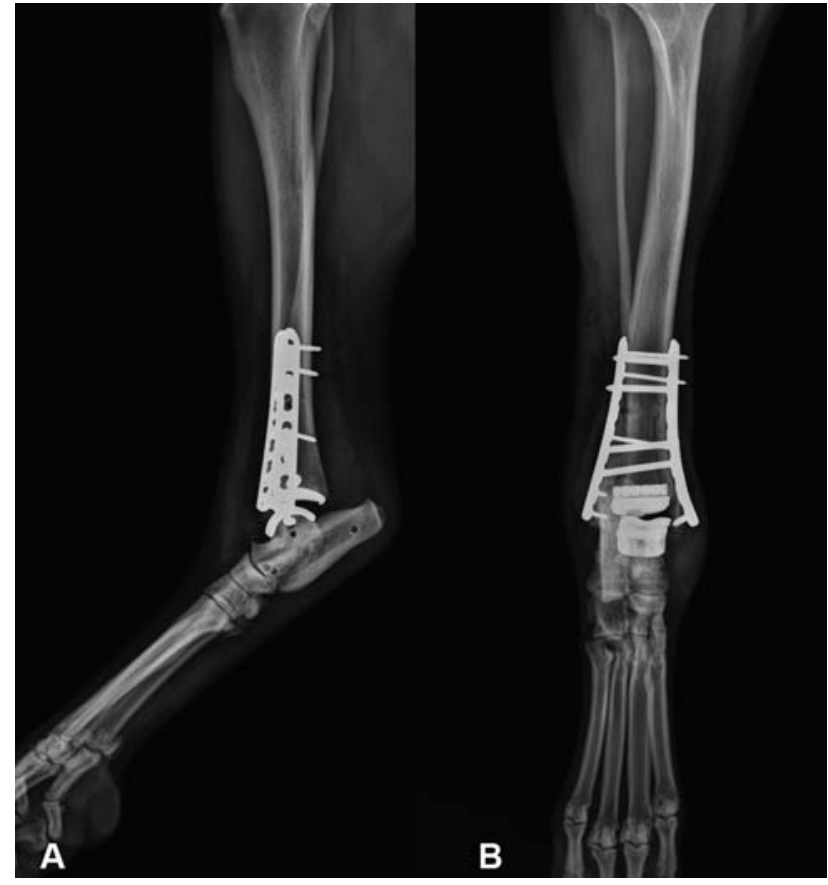

Fig. 7 Immediate (A) mediolateral and (B) caudocranial postoperative radiographs.

The dog was weight bearing on the operated limb the day following surgery and was discharged on day 3 postoperatively. Cage rest was instigated for 6 weeks with short lead walks for toileting purposes only. Skin sutures were removed 14 days postoperatively and at that time both medial and lateral surgical wounds had healed uneventfully.

The dog presented for re-examination 6 weeks postoperatively on no medication. The owner reported no concerns postoperatively during this period. On examination, the dog was $2 / 10$ th $^{11}$ lame on the left pelvic limb when walking. Talocrural range of motion was 80 degrees flexion, 160 degrees extension as assessed with goniometry. Soft tissue thickening of the joint was present and of similar magnitude to preoperatively. Palpation of the joint revealed no evidence of crepitus or apparent pain. The dog was sedated with medetomidine $(10 \mu \mathrm{g} / \mathrm{kg}$ IV) and butorphanol $(0.2 \mathrm{mg} / \mathrm{kg}$ IV). Radiographs were performed (-Fig. 8) that revealed the malleolar osteotomy had healed; subjectively there was further progression of tibiofibular arthrodesis with trabecular bone associated with the entire length of the tibial component and there was no lucency around either component. The dog was discharged with the recommendation to begin short lead walks of 10 minutes twice daily for a week, increasing by 5 minutes per week thereafter for a 6 week period.

Telephone conversation with the owner at 3 months postoperatively revealed the dog was on no medication, subjectively sound and being exercised on a flexi-lead for 45 minutes twice daily. The recommendation was made to incrementally reintroduce off lead activity back to normal levels over the following 8 weeks. The dog presented for reexamination 4 months postoperatively on no medication, and was being exercised off the lead for 45 minutes twice

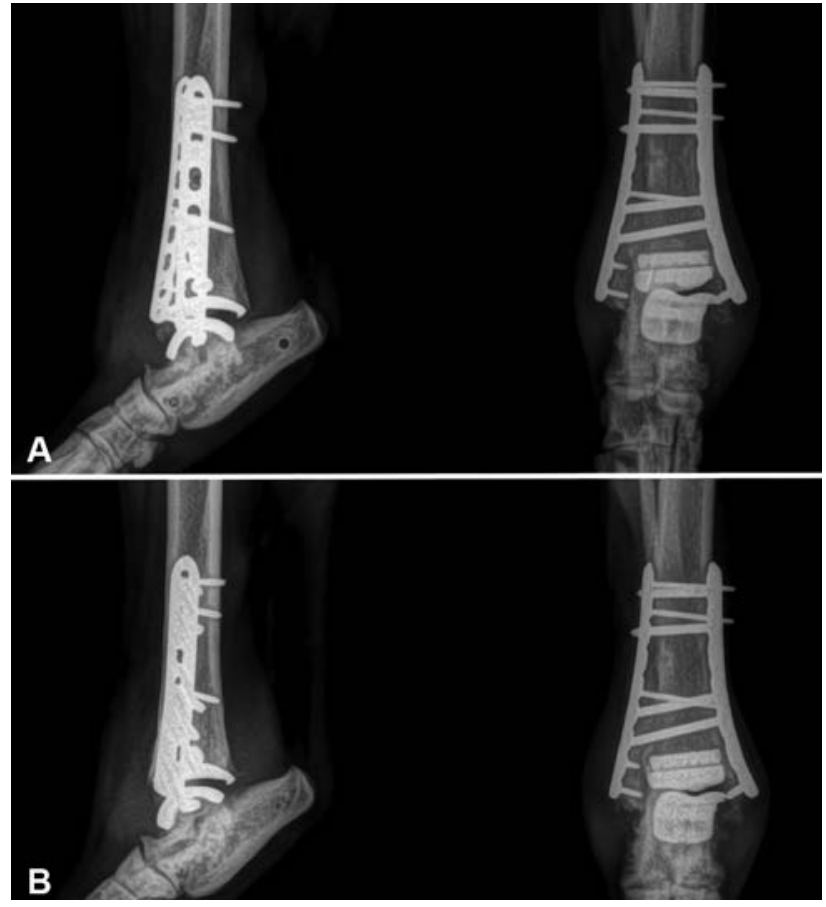

Fig. 8 (A) Mediolateral and caudocranial 6 weeks postoperative radiographs. (B) Mediolateral and caudocranial 4 months postoperative radiographs.

Table 1 Liverpool osteoarthritis in dogs (LOAD) scores for the patient

\begin{tabular}{|l|l|l|}
\hline Period of evaluation & $\begin{array}{l}\text { LOAD } \\
\text { score }\end{array}$ & $\begin{array}{l}\text { Interpretation- } \\
\text { severity of articular } \\
\text { disorder }\end{array}$ \\
\hline Preoperative & $25 / 52$ & Severe \\
\hline 6 weeks postoperative & $3 / 52$ & Mild \\
\hline 4 months postoperative & $5 / 52$ & Mild \\
\hline 6 months postoperative & $6 / 52$ & Mild \\
\hline
\end{tabular}

daily. On examination, the dog was subjectively sound when walking and trotting. Talocrural range of motion was 95 degrees flexion, 160 degrees extension as assessed with goniometry. Palpation of the joint revealed no evidence of crepitus or apparent pain. Radiographs were performed with sedation as previously described (-Fig. 8B) that had the same bone and component appearance to those taken at 6 weeks postoperatively.

Telephone conversation with the owner 6 months postoperatively revealed no concerns; the dog was still on no mediation, subjectively sound and exercise consisted of 90 minutes off-lead in the morning and 30 minutes on the lead in the afternoon.

\section{Questionnaires}

Liverpool Osteoarthritis in Dogs questionnaires ${ }^{15}$ were completed prospectively by the owner prior to surgery, 6 weeks and 4- and 6 months following surgery. The total score for these questionnaires at each visit is shown in - Table $\mathbf{1}$. 


\section{Discussion}

This case report is a first description of distal tibiofibular arthrodesis in the dog and the first published data on shortterm outcome following BCTAR.

The patient described herein did not have an osteochondral fragment identified either on CT or at the time of surgery; this corroborates with previous findings in $8 \%$ of patients with talar osteochondrosis. ${ }^{16}$ As no fragment was identified and as there is evidence that arthroscopic debridement of the joint in young dogs may not be synonymous with good outcome, ${ }^{16}$ arthroscopic evaluation of the joint was not pursued in this case.

Marked preoperative periarticular fibrosis was evident in this dog preoperatively, especially medially, as has previously been documented. ${ }^{17} \mathrm{~A}$ reduction in joint flexion was observed postoperatively as has similarly been observed following talar osteochondral fragment removal. ${ }^{17}$ The development of postoperative fibrosis following the extensive medial soft tissue dissection required for arthroplasty likely explains the reduction in range of motion observed.

The distal canine fibula articulates with the tibia via a small synovial cavity with an extensive tibiofibular syndesmosis. ${ }^{18}$ Cranial and caudal tibiofibular ligaments cumulatively afford low rotational mobility to the tibiotarsal joint. ${ }^{19}$ However, micro-movement, by the nature of a fibrous joint at this site, is expected to be present. To the authors' knowledge, canine distal tibiofibular arthrodesis has not been described in the literature. However, in man it has been used in the treatment of distal tibial osteochondroma, ${ }^{20}$ chronic inferior tibiofibular joint instability ${ }^{21}$ and is also essential in the support of the tibial component in some designs of humans ankle arthroplasty $^{22}$; an 8.5-fold increase in the risk of tibial component migration, having been reported if tibiofibular syndesmotic fusion, is not achieved. ${ }^{23}$ The use of this technique in man to provide appropriate support to the tibial component in ankle arthroplasty was the rationale for use in this dog. To proceed with arthroplasty in our case, there was a requirement to cross the tibiofibular joint space and mill away fibular bone to place a tibial component that was otherwise oversized, relative to the width of the tibia. Tibiofibular arthrodesis maximized the amount of confluent trabecular bone supporting the tibial component. If the lateral portion of the tibial component was not supported by bone or micromovement of the fibular relative to the tibia occurred, this could theoretically predispose to osteolysis around the tibial component with fibular fracture or aseptic loosening as possible sequalae. In addition to arthrodesis, we elected to plate the lateral aspect of the distal fibular to minimize the risk of fibular fracture occurring at the level of the tibial component where the fibular bone had been milled and the shelf of fibular bone remaining to support the tibial component laterally was thinnest.

Postoperative craniocaudal radiographs suggest the resultant joint space to be marginally wider on the medial aspect. This could be due to the extensive soft tissue dissection associated with the surgical approach medially, resulting in reduced medial periarticular soft tissue support; both long and short collateral ligament integrity were not com- promised by the dissection, based on palpation of the joint at the end of surgery. Alternatively, following placement of the COR pin in the talus and application of the alignment plate over the COR pin, if the medial joint space had been inadvertently 'closed' more than was necessary prior to application of the tibial fixation pins, this would have pulled the talus and pes into a mild varus, resulting in milling more bone off of the medial talus. Following implantation of the prosthesis and reduction in the malleolar osteotomy, this could result in a marginally wider joint space medially. The long-term effect of any discrepancy in joint space on component wear and general wear of this prosthesis system is currently unknown.

The data from the case report described herein must be interpreted in the context of the 6-month follow-up available. Long-term data on clinical performance, at the time of writing, are not available.

In summary, distal tibiofibular arthrodesis in concert with fibular plating permitted placement of an oversized talocrural implant in this patient with good clinical function. This technique may permit use of this arthroplasty system in otherwise undersized patients, until such a time that smaller implants become available from the manufacturer.

\section{Authors' Contributions}

N.J.B. and M.K. both performed the surgery described in this case report, drafted the manuscript, and approved the final version to be published. N.J.B. produced the figures unless otherwise stated.

\section{Conflict of Interest}

None declared.

\section{Acknowledgments}

The authors are grateful to BioMedtrix for granting permission to use several of the figures in this case report.

\section{References}

1 Marks RM. Mid-term prospective clinical and radiographic outcome of a modern fixed bearing total ankle arthroplasty. J Foot Ankle Surg 2019;58(06):1163-1170

2 Saltzman CL, Zimmerman MB, O'Rourke M, Brown TD, Buckwalter JA, Johnston R. Impact of comorbidities on the measurement of health in patients with ankle osteoarthritis. J Bone Joint Surg Am 2006;88(11):2366-2372

3 Piriou P, Culpan P, Mullins M, Cardon JN, Pozzi D, Judet T. Ankle replacement versus arthrodesis: a comparative gait analysis study. Foot Ankle Int 2008;29(01):3-9

4 Haddad SL, Coetzee JC, Estok R, Fahrbach K, Banel D, Nalysnyk L. Intermediate and long-term outcomes of total ankle arthroplasty and ankle arthrodesis. A systematic review of the literature. J Bone Joint Surg Am 2007;89(09):1899-1905

5 Stengel D, Bauwens K, Ekkernkamp A, Cramer J. Efficacy of total ankle replacement with meniscal-bearing devices: a systematic review and meta-analysis. Arch Orthop Trauma Surg 2005;125 (02):109-119

6 Slater MR, Scarlett JM, Donoghue S, et al. Breed, gender and age risk factors for canine osteochondrosis dissecans. Vet Comp Orthop Traumatol 1991;4:100-106

7 LaFond E, Breur GJ, Austin CC. Breed susceptibility for developmental orthopedic diseases in dogs. J Am Anim Hosp Assoc 2002; 38(05):467-477 
8 Fitzpatrick N. Arthrodesis verses novel joint replacement of the shoulder, elbow, stifle and hock in cats and dogs. In: Proceedings 16th ESVOT Congress, September 12-15, 2012, Bologna, Italy. pp 164-166

9 Acker RL. Tarsal joint replacement. In: Proceedings of the BioMedtrix 2018 Total joint conference, May 18-19, 2018; Hyatt Rosemont at Chicago O'Hare, Chicago, USA. pp 42-43

10 Van Der Meulen G. Design Rationale / System Overview. In: Proceedings of BioMedtrix TATE Elbow ${ }^{\mathrm{TM}}$ Workshop; 25-26th April 2009; Glasgow, UK

11 Sumner-Smith G. Gait Analysis and Orthopedic Examination. In: Slatter DS, ed. Textbook of small animal surgery 2nd ed. Philadelphia: WB Saunders; 1993:1578

12 Jaegger G, Marcellin-Little DJ, Levine D. Reliability of goniometry in Labrador Retrievers. Am J Vet Res 2002;63(07):979-986

13 Piermattei DL, Johnson KA. Approach to the wing of the ilium and dorsal aspect of the ilium. In: An Atlas of Surgical Approaches to the Bones and Joints of the Dog and Cat. 4th edition. Philadelphia: W.B. Saunders; 2004:278-281

14 Piermattei DL, Johnson KA. Approach to the late and medial malleolus and talocrural joint. In: An Atlas of Surgical Approaches to the Bones and Joints of the Dog and Cat. 4th edition. Philadelphia: W.B. Saunders; 2004:375-379

15 Walton MB, Cowderoy E, Lascelles D, Innes JF. Evaluation of construct and criterion validity for the 'Liverpool Osteoarthritis in Dogs' (LOAD) clinical metrology instrument and comparison to two other instruments. PLoS One 2013;8(03): e58125
16 Gielen I, van Bree H, Van Ryssen B, De Clercq T, De Rooster H. Radiographic, computed tomographic and arthroscopic findings in 23 dogs with osteochondrosis of the tarsocrural joint. Vet Rec 2002;150(14):442-447

17 van der Peijl GJW, Schaeffer IGF, Theyse LFH, Dijkshoorn NA, Schwencke M, Hazewinkel HA. Osteochondrosis dissecans of the tarsus in Labrador Retrievers: clinical signs, radiological data and force plate gait evaluation after surgical treatment. Vet Comp Orthop Traumatol 2012;25(02):126-134

18 Evans HE. Miller's Anatomy of the Dog. 3rd edition. Philadelphia, PA: Saunders-Elsevier; 1993:252

19 Barnett $\mathrm{CH}$, Napier JR. The rotatory mobility of the fibula in eutherian mammals. J Anat 1953;87(01):11-21

20 Ribbans WJ, Chadwick J, Natarajan R. Bilateral arthrodesis of the distal tibiofibular joint for deforming osteochondromatas. JRSM Open 2017; 8(08):2054270417718712https://journals.sagepub.com/doi/ 10.1177/2054270417718712 Accessed September 032020

21 Rafferty M, Al-Nammari SS, Sleat G, et al. Syndesmotic arthrodesis for chronic inferior tibiofibular joint instability. Orthop. Proc 2018; 96-B Supplement 17. Accessed September 03, 2020 at: https://online.boneandjoint.org.uk/doi/abs/10.1302/1358992X.96BSUPP_17.BOFAS2014-016

22 Barrow CR, Pomeroy GC. Enhancement of syndesmotic fusion rates in total ankle arthroplasty with the use of autologous platelet concentrate. Foot Ankle Int 2005;26(06):458-461

23 Pyevich MT, Saltzman CL, Callaghan JJ, Alvine FG. Total ankle arthroplasty: a unique design. Two to twelve-year follow-up. J Bone Joint Surg Am 1998;80(10):1410-1420 